\title{
Die Ko-Evolution von Wirtschaft und Wirtschaftsstrafrecht am Beispiel des Pharmamarketings
}

\author{
Ralf Kölbel und Nico Herold, Universität Bielefeld
}

\section{Einleitung}

Mit Blick auf die rechtliche Steuerung des Wirtschaftssystems fällt die Bestandsaufnahme traditionell ernüchternd aus. Die reale Normkonformität gilt ebenso wie die Kontrollwirksamkeit als defizitär. Zu erklären ist dieser Zustand mit der Eigenlogik juristischer und wirtschaftlicher Operationen, die in ,beiden Welten " für notorische Abstimmungs- und Anpassungsprobleme sorgt. Dem Einsatz von Strafrecht sind von daher massive Grenzen gesetzt - und zwar auch bei der Wahl neuartiger Implementierungswege („Compliance“). Dass die kriminalpolitische Euphorie, mit der die Herausbildung von wirtschaftseigenen Selbststeuerungsformen begleitet wird, deshalb bei näherem Hinsehen gedämpft werden muss, illustriert das Feld des sog. Pharmamarketings: Auf die neue Regulierungsanstrengung des (Straf-)Rechts reagieren die Wirtschaftsakteure mit Ausweichmanövern, was die ursprüngliche Funktionslogik aufrecht erhält.

\section{Autonomie von Wirtschaft und Recht}

Die grundsätzliche Limitierung (straf-)rechtlicher Steuerungsversuche der Wirtschaft tritt bei Zugrundelegung systemtheoretischer Annahmen besonders deutlich zutage $^{1}$. Im systemtheoretischen Gesellschaftsmodell stellen sowohl die Wirtschaft als auch das Recht (neben anderen gesellschaftlichen Subsystemen) eigenständige Funktionseinheiten dar, die jeweils nach einer eigenen Logik operieren. So besteht das Rechtssystem aus der fortwährenden Abfolge aneinander anknüpfender, rechtlicher Sprach- und Schreibakte, die die sozialen Ereignisse als Recht oder Unrecht thematisieren. Auf diese Weise sorgt es für die Stabilisierung eines Teils sozialer, eben rechtlicher Normen. Allein jene Kommunikationen, die in dieser spezifischen Art der Weltbetrachtung (im Lichte des „Rechtscodes“) erfolgen, sind Rechtskommunikationen und verkörpern die separierte „Gesellschaftseinheit" des Rechts. Dagegen bilden sämtliche Kommunikationen außerrechtlichen Inhalts dessen Umwelt. Das Wirtschaftssystem verarbeitet soziale Ereignisse etwa nach den Basiskriterien von Profit und Verlust.
Da sich die Operationen in den gesellschaftlichen Systemen nach jeweils eigenen Leitgrößen vollziehen, verlaufen sie gewissermaßen in einer jeweils anderen „Sprache“. Genau diese kommunikative Verschiedenheit markiert die Differenz der Systeme und deren Autonomie. Ereignisse in einem System lösen deshalb in einem anderen System, wo sie gar nicht „verstanden“ werden können, keine direkten Reaktionen aus. So haben beispielsweise wirtschaftliche Ereignisse im Rechtssystem keine unmittelbaren Folgen. Sie müssen dort vielmehr erst registriert und in eine rechtliche Konstruktion (einen „Rechtsfall“) „übersetzt“ werden, weil erst dies sie zu einem juristisch kommunizierbaren Element des Rechtssystems macht ${ }^{2}$. Wirtschaftliche Transaktionen, bei denen dies nicht geschieht, verlaufen am Rechtssystem dagegen vorbei und bleiben ein unverständliches „Rauschen“3.

Weitgehend autonom verläuft deshalb auch die Weiterentwicklung („Evolution“) der Systeme. Was hierbei stattfindet, ist stets eine systeminterne Selbst-Modifikation ${ }^{4}$ : Die Operationen in Recht oder Wirtschaft bringen dabei („zufällig“) eine neue Kommunikationsform hervor (eine Neuregelung oder Neuauslegung bzw. eine neue Geschäftsidee), die an die bisherigen Kommunikationen anschlussfähig ist und sich dadurch etabliert. Maßgeblich für ihren Erfolg ist aber ausschließlich die Eignung anhand der jeweils systemeigenen Kriterien. So verstetigt sich etwa eine neue Rechtsprechung nur, wenn sie sich dafür rechtsdogmatisch eignet - und nicht etwa wegen ihrer wirtschaftlichen (moralischen, sozialpolitischen usw.) Implikationen. In welche Richtung sich die Systeme entwickeln, bestimmt sich deshalb allein durch ihre autonome Logik. Auch die Geschwindigkeit, in der sie sich verändern, variiert mit den systemspezifischen Strukturen. Die moderne Gesellschaft wandelt sich somit in einer sich wechselseitig „irritierenden “, im Grunde aber eigenständigen Ko-Evolution ihrer Funktionssysteme. Eine abgestimmte Entwicklung von Wirtschaftsstrafrecht und Wirtschaft ist von daher kaum zu erwarten - wofür das sog. Pharmamarketing aktuelles Anschauungsmaterial bietet.

\section{Pharmamarketing als Wirtschaftsprozess}

Pharmahersteller sind wie jede andere wirtschaftliche Einheit der Gewinnerzielung verpflichtet. Die Erzeugung medizinisch besonders wirksamer Produkte ist ein probates Mittel zu diesem (!) Zweck (weil damit die Absatzaussichten steigen $)^{5}$, aber es ist nicht das einzige Mittel. Das Umsatzvolumen lässt sich auch mit arrivierten oder Nachahmerwirkstoffen (Analog-/,,me too-Präparate“) erreichen, sofern man deren Vertrieb in anderer Weise forciert. Aus diesem Grund investiert die Pharmaindustrie enorme Anstrengungen in den Marketingsektor und wendet hierfür bis zu $40 \%$ ihres Gesamtumsatzes auf (weit mehr als ihre Forschungsausgaben $)^{6}$. Bei der Arzneimittelwerbung der Industrie handelt es sich deshalb - anders als bei der pharmakologischen Forschung - nicht etwa um Wissenschaftskommunikation, sondern um Transaktionen im Wirtschaftssystem ${ }^{7}$, die in der dafür charakteristischen Profit- und Verlustlogik verlaufen. Sichtbar macht dies nicht zuletzt eine Reihe gut dokumentierter Marketingtechniken:

Besondere Bedeutung haben hierbei sog. Anwendungsbeobachtungen (AWB). Dabei erfasst der Arzt systematisch die therapeutischen Erfahrungen, die er mit einem zugelassenen Arzneimittel macht, um dessen Alltagsbewährung für den Hersteller zu dokumentieren (vgl. zu diesem eigentlichen Zweck \4 Abs. 23 S. 3 AMG). Solche AWB sind ausgesprochen verbreitet ${ }^{8}$. Nicht selten zahlen die Produzenten den Ärzten dabei allerdings ein Honorar, das den Wert des Dokumentationsaufwandes klar übersteigt. Dies soll primär auf das Verschreibungsverhalten einwirken und den Arzt motivieren, das fragliche Medikament möglichst oft einzusetzen. Angeblich dienen ca. $80 \%$ aller AWB - ungeachtet eines Verbotes $(\mathbb{S} 67$ Abs. 6 S. 3 AMG) - primär dem Vertrieb 9 Solche Schätzungen werden durch Studien gestützt, nach denen es AWB in der Regel an wissenschaftlichen Ansprüchen mangelt ${ }^{10}$. Kennzeichnend sind ferner die überdurchschnittlichen Verabreichungskosten von AWB-Medikamenten ${ }^{11}$. 
Dass sich auch die öffentliche Mitteilung über die Präparatwirksamkeit an der Absatzökonomie orientiert, spiegelt sich in den Einflussnahmen der Industrie auf einschlägige Arzneimittelstudien wider. Als aufschlussreich kann insofern ein eindeutiger Publikationsbias gelten: So besteht ein positiver Zusammenhang zwischen der Finanzierung von Untersuchungen und Ergebnissen, die für den pharmazeutischen Sponsor vorteilhaft $\operatorname{sind}^{12}$. Von der US-Pharmaindustrie gesponserte Forschung führt im Vergleich zu unabhängigen Studien mit einer deutlich angehobenen Wahrscheinlichkeit zu positiven Ergebnissen für das Produkt ${ }^{13}$. Autoren industriell finanzierter Forschungsarbeiten empfehlen die evaluierten (Unternehmens-)Medikamente mit sehr viel größerer Häufigkeit ${ }^{14}$. Über die Finanzierung hinaus werden weitere Einflussnahmen auf das Zustandekommen der Studien berichtet: unvollständige Registrierung der Studieninformationen, Verschweigen unerwünschter/ nachteiliger Arzneimittelwirkungen bis hin zur Verschleierung von Sterblichkeitsrisiken, vertragliche Einschränkung der Publikationsrechte, Einsatz von Ghostwritern und Gastautoren ${ }^{15}$.

Die am weitesten verbreitete Form des Pharmamarketings besteht jedoch nach wie vor in der direkten Beziehung zu niedergelassenen oder Krankenhausärzten. Etwa 77 \% der deutschen Ärzte werden mindestens wöchentlich von einem Pharmavertreter konsultiert ${ }^{16}$. Nach Hochrechnungen absolvieren die 15.000-20.000 Pharmareferenten jährlich 20 Mio. Praxis- oder Klinikbesuche. Dies alles dient hauptsächlich dazu, das Verschreibungsverhalten zugunsten der eigenen Produkte zu beeinflussen ${ }^{17}$ oder die jeweiligen Ärzte gar als „inoffizielle“ Fürsprecher zu gewinnen ${ }^{18}$. Dabei wird versucht, die persönliche Zugänglichkeit und die individuellen Vorlieben zu eruieren ${ }^{19}$ oder bei den Ärzten ein Verpflichtungsgefühl aufzubauen ${ }^{20}$. Bisweilen bedient man sich dabei eines Kategoriensystems, das Ärzte nach Lukrativität und Beeinflussbarkeit gruppiert. Um Umgang und Zuwendungen individuell zuschneiden zu können, werden persönliche Interessen, Hobbys, Persönlichkeitsmerkmale und die familiären Situationen erfasst ${ }^{21}$ und entsprechend angepasste Vorteile gewährt. Dies geht von Büroartikeln, über Produkt-Muster, Einladungen zu Produkteinführungen und Symposien oder Fortbildungsveranstaltungen bis hin zu Konferenz-Paketen. Der „return on investment“ wird von den Unternehmen dann in Datendanken (,prescription tracking“) kontrol- liert ${ }^{22}$. Dass die Maßnahmen das Verschreibungsverhalten tatsächlich beeinflussen, ist im Übrigen empirisch belegt. Die Verschreibungswahrscheinlichkeit steigt allein schon durch bloße Vertreterbesuche, und zwar unabhängig von Zuwendungsform oder - wert $^{23}$. Fortbildungen zu Produkten führen zu deren verstärkter Verordnung ${ }^{24}$. Die Mehrheit der Ärzte ist sich gleichzeitig der Marketingabsichten durchaus bewusst ${ }^{25}$, wobei sie freilich eine Beeinflussungsgefahr nur bei ihren Kollegen verorten und nur selten auch bei sich selbst ${ }^{26}$.

\section{Die Veränderungen im (Straf-) Recht}

\section{Systeminterne Debatten}

Die geschilderten Methoden konnte die Pharmaindustrie jahrelang (straf-)rechtlich nahezu unbehelligt anwenden. Dies änderte sich jedoch, als das Rechtsystem insbesondere die Marketingmaßnahmen gegenüber Ärzten aufzugreifen begann. Auslöser für diesen neuen innerrechtlichen Entwicklungsprozess war allerdings nicht etwa der typische Anlass, bei dem eine (rechtlich fragwürdige) Transaktion im Wirtschaftssystem vor Gericht gelangt und dann neuartige Rechtsbehauptungen evoziert. Vielmehr wurde die Debatte systemintern durch den rechtswissenschaftlichen Diskurs ausgelöst.

Ursprünglich hatte die juristische Kommunikation die Einordnung des Vertragsarztes als sog. „Beauftragten“ der Kassen einhellig abgelehnt. Diese Sichtweise wurde plötzlich („zufällig“) auf den Prüfstand gestellt und es wurden die oben geschilderten Verschreibungsanreize mit einer Bestechung i.S.v. \ 299 StGB assoziiert ${ }^{27}$. Den Anstoß für diese Neueinordnung bildete eine kurz davor ergangene BGH-Entscheidung, die den Vertragsarzt zum „Vertreter“ der Kassen bei der Arzneimittelversorgung erklärte ${ }^{28}$. Freilich verhielt sich der rechtsdogmatische Diskurs, auch nachdem die neue Rechtsdeutung als prinzipiell fortentwicklungsfähig eingeführt worden war, zunächst noch ablehnend (sowohl im strafrechtlichen Schrifttum $^{29}$ wie auch der justiziellen Praxis ${ }^{30}$ ). Dies änderte sich aber, als die Kommentarliteratur die Anwendbarkeit des $\$ 299$ StGB auf das Pharmamarketing zu befürworten begann ${ }^{31}$. In der Folge wurde dies nicht nur zur vorherrschenden Wissenschaftsmeinung ${ }^{32}$, sondern Ende 2010 auch von der Justiz akzeptiert. Im Anschluss an das OLG Braunschweig sahen nunmehr auch andere Gerichte im Vertragsarzt (als Marketingadressat) einen Beauftragten ( $\$ 299$
I StGB) und/oder Amtsträger ( $\mathbb{S} 331 \mathrm{StGB}$ ) und behandelten auf dieser Basis die oben geschilderte AWB- und Zuwendungspraxis als strafbare Korruption ${ }^{33}$.

Gegenwärtig stabilisiert sich diese Strafrechtsentwicklung systemintern also als herrschende Meinung. Die juristische Innovation setzt sich voraussichtlich durch - dies aber in genau der oben (II.) skizzierten Verlaufsautonomie: d.h. nicht etwa wegen ihrer kriminalpolitischen Aktualität als Antwort auf einen gesellschaftlich markierten Missstand, sondern ausschließlich wegen der strafrechtlichen Kompatibilität („Begründbarkeit") des neuen rechtsdogmatischen Konzepts. Demgemäß sind moralische, medizinische oder wirtschaftliche Fragen für die strafrechtliche Behandlung des Pharmamarketings völlig unerheblich geblieben.

\section{Politisch ausgelöste Normentwicklung}

Parallel zu dieser Rechtsprechungsentwicklung werden durch die Methoden der Pharmaindustrie auch legislative Reaktionen angeregt. Eine solche Rechtsevolution, die durch Gesetzgebung erfolgt, wird allerdings im geringeren Maße als der judikative Rechtswandel von den systeminternen Rechtsfaktoren bestimmt. Bei ihr determinieren anstelle der juristischen Passfähigkeit vielmehr die politischen Gegebenheiten, welche Regelungsvorschläge (Variationen) das Gesetzgebungsverfahren erfolgreich durchlaufen (Selektion). So gehen Gesetzesinitiativen vielfach darauf zurück, dass Gesellschaftszustände zum Gegenstand der medialen und gesellschaftlichen Kommunikation avancieren und sich aus deren Regulierung politisches Kapital schlagen lässt. Welche Regelungsoptionen sich durchsetzen können, ist ebenfalls durch politische Konstellationen bedingt (Machtverhältnisse, Koalitionen, Lobby- und Unterstützungshintergründe). Erst in der dritten Evolutionsphase (Restabilisierung) kommt das Rechtssystem wieder prägend zum Zuge. Dann sind es die gerichtlichen und dogmatischen Diskurse, die mit ihrer Interpretationsleistung in den Entscheidungsreihen der Fallpraxis den Effektivbereich der Neuregelung konstituie$\operatorname{ren}^{34}$.

Dieser Abfolge entsprechend reagierte das politische System, als die Medien diverse korruptionsnahe Kooperationsformen im Gesundheitssystem skandalisierten (Signalworte zwischen 2008 und 2010 waren: Zuweisungsvergütung, Einweiserpauschale, Kopfgeld, der gekaufte Patient ${ }^{35}$. Darauf- 
hin wurde im Verlauf eines kaum zu rekonstruierenden Gesetzgebungsprozesses der \128 SGB V mehrfach „reformiert" ${ }^{36}$ und dabei u.a. um einen 6. Absatz ergänzt, der das Verbot verschreibungsbezogener $\mathrm{Zu}$ wendungen auf pharmazeutische Unternehmen, Apotheken und Großhändler erstreckt (sowohl untereinander als auch gegenüber Ärzten). Diese Vorschrift gelangte daraufhin zurück in den rechtlichen Diskurs und unterliegt dort nunmehr den üblichen Auslegungs- und Sinngebungsversuchen. Angesichts eines breiten Anwendungsbereiches (der theoretisch bis zu 5.040 ZuwendungsKombinationen erfassen kann) sowie einer erheblichen Unbestimmtheit in den tatbestandlichen Details (etwa hinsichtlich der Einbeziehung von AWB) besteht hierfür Anlass genug ${ }^{37}$. Welche Konturen das Zuwendungsverbot in $\$ 128$ VI SGB V nach diesen Interpretationsdiskursen annehmen und in welcher Rigorosität es mitsamt seiner außerstrafrechtlichen Sanktionen implementiert werden wird, ist dem politisch generierten Normtext derzeit also noch nicht anzusehen. Dass die Stoßrichtung sich aber mit der strafrechtlichen Korruptionsrechtsprechung deckt, steht freilich fest.

\section{Die Reaktion der Wirtschaft}

Solche Veränderungen im Recht vollziehen sich unabhängig von Wirtschaftssystem - wirken in dieses aber auch nur mittelbar durch. Aus systemtheoretischer Warte nimmt die Wirtschaft den Wandel im Rechtssystem zwar wahr, beachtet sie aber nicht ihrer SollGeltung wegen und passt sich daran auch nicht wunschgemäß an. Vielmehr greift sie jede Norm in ihren Transaktionskalkülen prinzipiell nur als eine „von Entdeckungswahrscheinlichkeit und Sanktionsintensität abhängige Kostenirritation “ auf ${ }^{38}$. Untersagte oder grenzwertige Aktivitäten unterbleiben deshalb lediglich dort, wo dies ökonomisch sinnvoll erscheint, insbesondere wegen fehlender Vorteile oder wegen drohender Deliktsbegehungskosten (strafrechtlicher Sanktionen, zivilrechtlicher Haftung, Imageschäden, Störungen in betrieblichen und geschäftlichen Beziehungen ${ }^{39}$. Da also das Wirtschaftssystem auch auf Rechtsevolutionen (d.h. auf jede Neuinterpretation und jedes neue Gesetz) allein über deren ökonomische Implikationen reagiert, ist es keineswegs ausgemacht, dass sich diese Reaktion in Ausmaß und Art mit den Intentionen in Politik und/oder Rechtssystem deckt.
Entsprechend verhält es sich auch bei der rechtlich und politisch angestoßenen, wirtschaftseigenen Selbststeuerung (wirtschaftseigene Kodizes und sog. Healthcare-Compliance). Deren offiziöse kriminalpolitische Funktion ist freilich klar. Sie soll im Wege der betrieblichen Selbstkontrolle für gesetzeskonformes Verhalten aller Mitarbeiter in der gesundheitswirtschaftlichen Branche sorgen $^{40}$. Vielfach handelt es sich bei der praktischen Einrichtung und Umsetzung de facto aber um eine "selective compliance“. Normkonformität wird dann nur dort hergestellt, wo dies ökonomisch sinnvoll oder wenigstens unschädlich ist, also beispielsweise bei den sog. occupational crimes. Im Hinblick auf rechtlich fragwürdige oder gar strafbare Wirtschaftspraktiken, die dem Unternehmen zum Vorteil gereichen (wie etwa die nunmehr illegalisierten Pharmamarketing-Maßnahmen), kann es dagegen ökonomisch sinnvoller sein, zwar die Reputationsvorteile von öffentlich vorzeigbaren Compliance-Systemen in Anspruch zu nehmen, aber die ursprünglichen Transaktionsformen wegen ihrer wirtschaftlichen Funktionalität beizubehalten und lediglich besser zu kaschieren oder in juristische Grauzonen zu verlegen. Dass dies tatsächlich geschieht, deuten aktuelle Entwicklungen durchaus an:

So wurden in der Industrie nicht nur die Kodizes der internationalen Wirtschaftsverbände ${ }^{41}$ und Verhaltensleitlinien der großen Arzneimittelhersteller erlassen ${ }^{42}$, sondern neben betrieblichen Compliance-Strukturen auch wirtschaftseigene Regelungswerke und Spruchkörper mit Sanktionskompetenz eingeführt ${ }^{43}$. Hinzuweisen ist hier insbesondere auf die Vereinigungen „Freiwillige Selbstkontrolle für die Arzneimittelindustrie e.V.“ (FSA) sowie „Arzneimittel und Kooperation im Gesundheitswesen e.V.“ (AKG), die neben Verhaltensvorgaben für die Mitgliedsunternehmen auch Verfahrungsordnungen und sanktionsbefugte Schiedsgerichte eingerichtet haben $(2004)^{44}$. All dies ging den oben skizzierten Rechtsentwicklungen freilich zeitlich voraus und verdankte sich ursprünglich dem Eigeninteresse der Wirtschaft an Wettbewerbsregeln auf dem Arzneimittelmarkt. Jetzt, da das Rechtssystem (gewissermaßen nachholend) den Bereich des Pharmamarketings als Unrecht etikettiert, konkretisieren diese Einrichtungen die neuen (straf-)rechtlichen Vorgaben. Zugleich wächst ihre Relevanz für die Reduzierung von Vertriebsrisiken. Das traditionelle Pharmamarketing ist nämlich nunmehr einer stärkeren Kostengefahr ausgesetzt. Ne- ben dem neuen „hard risk“ der straf- und sozialrechtlichen Sanktionierung entstehen die „soft risks“ einer „schlechten Presse“. Hierdurch wiederum drohen Reputationsschäden, Verschreibungsrückgänge oder ein abträgliches Partner-Profil (,value dossier“), das die Kassen bei der Vereinbarung von Rabattverträgen berücksichtigen könnten ${ }^{45}$.

Andererseits liefe eine vollständige und konsequente Implementierung der Kodizes - d.h. auch eine Orientierung der Vertriebspraxis an den neuen Kriterien des Rechts - auf eine Erschwerung der Arzneimitteldistributionen hinaus und hätte ihrerseits also ebenfalls wirtschaftlich nachteilige Implikationen ${ }^{46}$. Es liegt daher nahe, dass die Unternehmen eine Balance zwischen beiden Belangen suchen und im Sinne der „selective compliance" solche Marketing-Techniken entwickeln, die sich am Rande des ,gerade noch legal“ bewegen (oder die Grenzüberschreitung geschickter als vorher bemänteln). Eine solche taktierende Anpassung an die neuen Bedingungen - d.h. das Ausschöpfen des rechtlich verbliebenen Rahmens und die Nutzung von Grauzonen im Pharmavertrieb - werden im Feld demgemäß auch als „Herausforderung “ markiert ${ }^{47}$. Und in der Folge geht man dann tatsächlich zu Vertriebsfördermethoden über, die zwar prima vista (noch) legal sind, die aber die medizinischen Notwendigkeiten und die Patientenbedürfnisse den ökonomischen Absatzinteressen in gleichem Maße unterordnen, wie dies auch bei den inzwischen inkriminierten Marketingformen (oben II.) geschah ${ }^{48}$. So geraten in einem multidimensionalen Ansatz nicht nur wichtige Entscheidungsträger, Zulassungsbehörden, wirtschaftspolitische Institutionen und politische Parteien ${ }^{49}$, sondern vor allem auch Patienten und deren Organisationen ins Vi$\operatorname{sier}^{50}$. Solche neuen Strategien wollen den kranken Menschen für sich gewinnen und setzen darauf, dass dieser den Arzt am effektivsten zur Verschreibung der (,neuen“, „innovativen“ usw.) Arzneimittel des fraglichen Herstellers drängt ${ }^{51}$. Nicht selten werden hierbei auch neue Krankheiten erfunden („desease mongering“). Gerechtfertigt wird die vertriebstaktische Umstellung von der push- auf die pull-Technologie mit der Verpflichtung, den „mündigen Patienten“ über Präparat- und Heilungschancen ins Bild setzen zu wollen ${ }^{52}$. Tatsächlich geht es aber auch hier nur um einen Wettbewerbsfaktor, zumal das Direct-to-Consumer Advertising effektiv wirkt: Patienten lassen sich von der Produktwerbung beeinflussen und erhalten von ihren Ärzten das Mittel ihrer Wahl ${ }^{53}$. 


\section{Ausblick}

Die parallele Entwicklung von Recht und Wirtschaft ist derzeit im Feld des Arzneimittelvertriebs ,in action“ und damit besonders eindrucksvoll zu ersehen: Etablierte Transaktionsformen des Pharmamarketings sind als Teil des Wirtschaftssystems hochprofitabel und befinden sich samt den zugehörigen Selbstkontrolltechniken in einem ständigen Perfektionierungsprozess. Dies blieb bisher rechtlich und politisch weitestgehend unangetastet. Angestoßen von medialer Skandalisierung geht man nun allerdings von politischer Seite dazu über, Pharmamarketing regulieren zu wollen. Parallel dazu vollzieht sich dessen Kriminalisierung im Strafrecht. Dass nun aber die Pharmabranche die in Frage stehenden Marketingmethoden aufgibt oder auf ein tolerierbares Maß reduziert, ist aus Gründen der Konkurrenzfähigkeit nicht zu erwarten. Deshalb fängt man die rechtlichen/politischen Anstöße durch die geringstmöglichen Prozessmodifikationen auf. So reagiert die Wirtschaft allenfalls dort mit (Rechts-)Konformität, wo sich keine risikoarme und ökonomisch sinnvolle Minder-Anpassung findet - also vor allem im Bereich der leicht aufzudeckenden Praktiken (wie voraussichtlich vor allem bei der traditionellen Vorteilsgewährungen gegenüber zahlreichen niedergelassenen Ärzten). Ansonsten greifen auch die Erwartungen an die Wirksamkeit von Compliance-Systemen zu hoch. Die zu beobachtenden Reaktionen der Pharmabranche illustrieren die Gefahr, dass die Compliance-Bewegung als bloßes „window-dressing " fungiert, während die wirtschaftliche Praxis die rechtlich problematischen Vertriebsmuster aufrechterhält oder durch widerstandsärmere (aber nicht minder fragwürdige) funktionelle Äquivalente ersetzt. Der politisch und/oder juristisch intendierte Steuerungseffekt bleibt deshalb aus. Es wird allenfalls ein „Innovations-Druck“ auf die Wirtschaft erzeugt, der womöglich die Entwicklung rechtlich wesentlich schwerer zu handhabender Kooperationsformen fördert. Sofern Recht und Wirtschaft also überhaupt so etwas wie eine gemeinsame Grundrichtung („structural drift“) einschlagen, geschieht dies jedenfalls nicht im Sinne einer „Disziplinierung“ des Arzneimittelvertriebs.

Prof. Dr. Ralf Kölbel ist Mitglied im Institut für interdisziplinäre Konflikt- und Gewaltforschung (IKG) und im Institut für Rechtstatsachenforschung und Rechtspolitik der Universität Bielefeld.
Literaturverzeichnis

Abegg, A. (2006). Evolutorische Rechtstheorie, in: Buckel/Christensen/Fischer-Lescano (Hrsg.), neue Theorien des Rechts, Stuttgart 2006

Als-Nielsen, B., Chen, W., Gluud, C. et al. (2003): Association of Funding and Conclusions in Randomized Drug Trials. A Reflection of Treatment Effect or Adverse Events? Journal of the American Medical Association 290, S. 921-928

Bauer, H. H., Hahn, O. K., Hammerschmidt, M. (2006): Patientenbindung durch Kommunikation. Impulse für das Pharmamarketing. Wissenschaftliches Arbeitspapier W108 Mannheim

Boers, K., Theile, H., Karliczek; K. M. (2003): Wirtschaft und Strafrecht - Wer reguliert wen? Kölner Zeitschrift für Soziologie und Sozialpsychologie 43, S. 469-493

Bowman, M. A., Pearle; D. L. (1998): Changes in Drug Prescribing Patterns Related to Commercial Company Funding of Continuing Medical Education. The Journal of Continuing Education in the Health Profession 8, S. $13-20$

Brett, A. S., Burr W., Moloo, J. (2003): Are Gifts From Pharmaceutical Companies Ethically Problematic - A Survey of Physicians. Archives of Internal Medicine 163, S. 22132218

Burk, E. (2010): Zum Anwendungsbereich der Zuwendungs-und Beteiligungsverbote in \$128 Abs. 2 und 6 SGB V - Leitlinien für pharmazeutische Unternehmer und Hilfsmittelanbieter. Pharma Recht 3, S. 89-101

Campbell, E. G., Gruen, R.L., Mountford, J.et al. (2007): A National Survey of Physician-Industry Relationships. National English Journal of Medicine 356, S. 1742-1750

Caudill, T.S., Johnson, M.S., Rich, E.C et al. (1996): Physicians, Pharmaceutical Sales Representatives, and the Cost of Prescribing. Archives of Family Medicine 5, S. 201-206

Chren, M.-M., Landefeld, C. S. (1994): Physicians' Behavior and Their Interactions With Drug Companies. A Controlled Study of Physicians Who Requested Additions to a Hospital Drug Formulary. The Journal of American Medical Association 271, S. 684689

Dietrich, E.-S. (2009): Die meisten deutschen Anwendungsbeobachtungen sind zur Generierung wissenschaftlich valider Erkenntnisse nicht geeignet. PharmacoEconomics - German Research Articles 7/2009, S. 3-14

Ehlers, A. P.F. (2005): Pharma-Events und ärztliche Fortbildung. Individuelles Fortbildungssponsoring aus rechtlicher Sicht: Ist Selbstregulierung staatlicher Reglementierung überlegen? Pharma-Marketing-Journal 3/2005, S. 83-99

Fisher, M. A. (2003): Physicians and the pharmaceutical Industry. A dysfuncional relationship? Perspectives in Biology and Medicine 46 , S. 254-72

Fugh-Berman, A., Ahari, S. (2007): Following the Script: How Drug Reps Make Friends and Influence Doctors Public Library of Science Medicine 4, S. 621-625

Fulda, Ch. (2010): Alter Wein oder alles Essig? Zur Erstreckung von $\$ 128$ SGB V auf die Arzneimittelversorgung in der GKV. Pharma Recht 3, S. 94-101

Frister, H. (2010): Der Vertragsarzt als Beauftragter der Krankenkassen im Sinne des $\mathbb{S}$ 299 StGB?, in: Michael Lindemann/Rudolph Ratzel (Hrsg.): Brennpunkte des Wirtschaftsstrafrechts im Gesundheitswesen. Nomos, Baden-Baden 2010, S. 99 - 112

Gagnon, M.-A., Lexchin, J. (2008): The Cost of Pushing Pills: A New Estimate of Pharmaceutical Promotion Expenditures in the United
States. Public Library of Science Medicine 5, S. 29-33

Geis, M. (2005): Tatbestandsüberdehnungen im Arztstrafrecht am Beispiel "Beauftragtenbestechung" des Kassenarztes nach $\$ 299$ StGB. wistra 10, S. 369-371

Glaeske, G., Schubert, K. (2006): Einfluss des pharmazeutisch-industriellen Komplexes auf die Selbsthilfe. Universität Bremen - Zentrum für Sozialpolitik

Harms, F., Gänshirt, D., Lonsert, M. (2005): Zukunftsperspektiven für pharmazeutisches Marketing. Die Pharmazeutische Industrie 67, S. $865-870$

Hofer, P., Klümper, M. (2009): Der VDGH-Kodex. Ein neuer Stern am Kodexhimmel? Eine Betrachtung der aktuellen Kodexlandschaft der Medizinprodukteindustrie. Medizinprodukte Journal 16, S. 23-32

Jelinek, G., Neate, S. (2009): The influence of the pharmaceutical industry in medicine. The Journal of Law and Medicine 17, S. 216-23

Katz D., Caplan A.L., Merz J.F. (2003): All Gifts Large and Small. Toward an Understanding of the Ethics of Pharmaceutical Industry GiftGiving. The American Journal of Bioethics 3/2003, S. 39-48

Kaufmann, M., Voland, Th. (2011): "Ich war noch niemals in New York...". Die Auswirkungen des $\mathbb{} 128$ SGB V auf das Angebot von Schulungsmaßnahmen und sonstige Leistungen durch die pharmazeutische Industrie. Neue Zeitschrift für Sozialrecht, S. 281-284

Khanfar, N.M., Polen H.H., Clauson K.A. (2009): Influence on Consumer Behavior: The Impact of Direct-to-Consumer Advertising on Medication Requests for Gastroesophageal Reflux Disease and Social Anxiety Disorder. Journal of Health Communication: International Perspectives 14 , S. $451-460$

Klümper, M. (2009): Die Neuerungen an $\mathbb{1 2 8}$ SGB V durch die 15. AMG-Novelle. Alter Wein in neuen Schläuchen! Pharma Recht 31, S. 591-595

Klümper, M., Walther, H.-P. (2010): Auditierung und Zertifizierung von Pharmaunternehmen im Bereich Healthcare Compliance. Konzept und Chance eines innovativen ComplianceAnsatzes. Pharma Recht 32, S. 145-152

Klümper, M., Vollebregt, E. (2009): Business Compliance 2.0! Neue Compliance-Ansätze im Unternehmen zur Bewältigung kommender Herausforderungen. Pharma Recht 31, S. $319-320$

Klümper, M., Eggerts, O. (2010): Schutz vor staatlicher Strafe durch Selbstregulierung? Die Auswirkungen von Entscheidungen der Selbstregulierungsstellen auf Strafverfahren gegen Mitarbeiter der Pharmaindustrie. Pharma Recht 32, S. 13-18

Korzilius, H.; Rieser, S. (2007): Pharmaberater: Für manche Fachmann, für andere Buhmann

Deutsches Ärzteblatt 104, S. 156-161

Kölbel, R. (2008a): Wirtschaftskriminalität und unternehmensinterne Strafrechtsdurchsetzung. Monatsschrift für Kriminologie und Strafrechtsreform 91, S. 22-37.

Kölbel, R. (2008b): Zur wirtschaftsstrafrechtlichen Institutionalisierung des Whistleblowing. Juristenzeitung 63, S. 1134-1141

Kölbel, R. (2011a): Strafbarkeitsnahe vertragsärztliche Kooperationsformen In: Neue Zeitschrift für Strafrecht, S. 195 $-200$

Kölbel, R. (2011b): Korruptionsprävention im Gesundheitssystem durch Selbstkontrolle der Wirtschaft?, In: Duttge, G. (Hrsg.): Tatort Gesundheitsmarkt. Rechtswirklichkeit - Strafwürdigkeit - Prävention, Göttingen, S. $87-107$.

Kölbel, R., Herold, N. (2010): Whistleblowing. Eine kriminologische Analyse aus Anlass der 
aktuellen kriminalpolitischen Debatte. Monatsschrift für Kriminologie und Strafrechtsreform 93, S. 425-441

Kuhlen, L. (2010): Strafrecht und freiwillige Selbstkontrolle der Wirtschaft: das Beispiel der Pharmaindustrie. In: Festschrift für Winfried Hassemer. Felix Herzog, Ulfrid Neumann (Hrsg.), Heidelberg 2010, S. 875-889

Lexchin, J. (1993): Interactions between physicians and the pharmaceutical industry: What does the literature say? Canadian Medical Association 149, S. 1401-1407

Lieb, K., Brandtönies, S. (2010): Eine Befragung niedergelassener Fachärzte zum Umgang mi Pharmavertretern. Deutsches Ärzteblatt International 107, S. $392-398$

Luhmann, N. (1993): Das Recht der Gesellschaft. 1. Auflage. Frankfurt am Main. 1993

Luhmann, N. (1994): Soziale Systeme. 4. Auflage. Frankfurt am Main 1994

Moynihan, R. (2003): Who pays for the pizza? Redefining the relationships between doctors and drug companies. 1: Entanglement. British Medical Journal 326, S. 1189-1192

Oldani, M. J. (2004): Thick presciptions: Toward an Interpretation of Pharmaceutical Sales Practices. Medical Anthropology Quarterly 18. S. 325-356

Passarge, M. (2011): Aktuelle Entwicklungen und Tendenzen im Bereich Health Care Compliance - unter besonderer Berücksichtigung mittelständischer Unternehmen. Pharma Recht 33 S. 80 - 85

Pragal, O. (2005): Das Pharma-"Marketing” um die niedergelassenen Kassenärzte: "Beauftragtenbestechung” gemäß $\$ 299$ StGB! Neue Zeitschrift für Strafrecht 25, S. 133-136

Pragal, O., Apfel, H. (2007): Bestechlichkeit und Bestechung von Leistungserbringern im Gesundheitswesen. Arzneimittel und Recht 3, S. $10-20$

Randall, M., Rosenbaum, J., Rohrbaugh, R.M. et al. (2005): Attitudes and Behaviors of Psychiatry Residents Toward Pharmaceutical Representatives Before and After an Educational Intervention. Academic Psychiatry 29, S. 33-39

Sahan, O., Urban, K. (2011): Die Unbestechlichkeit niedergelassener Vertragsärzte. Zeitschrift für internationale Strafrechtsdogmatik 7, S. 23-28

Schott, G., Pachl, H., Limbach, U. et al. (2010a/2010b): Finanzierung von Arzneimittelstudien durch pharmazeutische Unternehmen und die Folgen. Deutsches Ärzteblatt International 107, S. 279-284 (Teil 1) und 295-301 (Teil 2).

Schneider, N., Lückmann, S. L. (2008): Pharmasponsoring in der ärztlichen Fort- und Weiterbildung. Zeitschrift für Allgemeinmedizin 84 , S. 516-524

Schneider, H. (2010): Strafrechtliche Grenzen des Pharmamarketings. Zur Strafbarkeit der Annahme umsatzbezogener materieller Zuwendungen durch niedergelassene Vertragsärzte. Höchst Richterliche Rechtsprechung im Strafrecht 11, S. 241-247

Teubner, G. (1989): Recht als autopoietisches System. Frankfurt am Main 1989

Teubner, G. (1999): Eigensinnige Produktionsregimes: Zur Ko-Evolution von Wirtschaft und Recht in den varieties of capitalism, in: Soziale Systeme 5, S. 7-25

Theile, H. (2008): Unternehmensrichtlinien - Ein Beitrag zur Prävention von Wirtschaftskriminalität? Zeitschrift für Internationale Strafrechtsdogmatik 4, 406-418

Theile, H. (2010): Strafrechtliche Hypertrophie und ihre Folgen. Das Beispiel der verfahrenserledigenden Urteilsabsprachen in Wirtschaftsstrafuerfabren. Monatsschrift für Kriminologie und Strafrechtsreform 93, S. $147-163$

Ulsenheimer, K. (2008): Arztstrafrecht in der Praxis. 4., neu bearbeitete und erweiterte Auflage. Heidelberg 2008

Weiss, H. (2008): Korrupte Medizin. 1. Auflage. Köln 2008

(Endnotes)

1 Dazu im Anschluss an Luhmannsche Konzeptionen und m.w.N. bereits KÖLBEL (2008a); (2008b); vgl. auch KÖLBEL / HEROLD (2010); THEILE (2010).

2 dazu LUHMANN (1993), S. $84 \mathrm{ff}$

3 vgl. LUHMANN (1994), S. 197.

4 Zum Folgenden näher LUHMANN (1993), 239 ff., 257 ff.; TEUBNER 1989, 66 ff.; ABEGG (2006), $378 \mathrm{ff}$

5 Etwa JELINEK / NEATE (2009), S. 1; FISHER (2003), S. $255 \mathrm{f}$

6 Vgl. für Ausgaben in den USA bei GAGNON / LEXCHIN (2008), S. 29 f.; sowie für den Anteil am Gesamtumsatz CAUDILL / JOHNSON ( RICH et al. (1996), S. 201).

7 So auch CAUDILL / JOHNSON / RICH et al. (1996), S. 201

8 Die genaue Häufigkeit ist nicht bekannt. Der Verband forschender Pharma-Unternehmen zählt gegenwärtig 256 freiwillig gemeldete Studien seiner Mitglieder (http://www.vfa.de/ de/arzneimittel-forschung/datenbanken-zu-arzneimitteln/nisdb - Aufruf vom 13.10.2011). Für 2008 ging die Kassenärztliche Bundesvereinigung (KBV) davon aus, dass Ärzte in 85.000 Fällen an 329 AWB zu 235 Präparaten teilgenommen haben (http://www.faz.net///Rub0E9EEF84AC1E4A389A8DC6C23161FE44/ Doc EBC0D6DC2B5A94E429815DEFF9CE8 575F ATpl Ecommon Scontent.html - Aufruf vom 13.10.2011).

9 So nach (http://www.versicherungsnetz.de/ news/Meldung.asp?Meldung=1494 - Aufruf vom 13.10.2011) die Schätzung der KBV in einer Pressemittelung vom 13.12.2002

10 Ausführliche Analyse bei DIETRICH (2009), S. $3-14$

11 DIETRICH (2009), S. 7

12 Vgl. die Metaanalyse bei SCHOTT / PACHL / LIMBACH et al. (2010b), S. 295 f., 299

13 Vgl. JELINEK / NATE (2009), S. 9 m.w.N.

14 So die Befunde bei ALS-NIELSEN / CHEN / GLUUD et al. (2003), S. 921 f. auch m.w.N.

15 Vgl. dazu die ausführliche Literatur-Übersicht bei SCHOTT / PACHL / LIMBACH et al. (2010a), S. 279 f. und DIES. (2010b), S. 299 Vgl. auch JELINEK / NATE (2009), S. 3 m.w.N.

16 Vgl. LIEB / BRANDTÖNIES (2010), S. 392, 394; KORZILIUS / RIESER (2007), S. 156 f. (mit Hinweisen zu den unterschiedlichen Besuchsmodalitäten). In den USA berichten sogar $94 \%$ der Ärzte von bestehenden Kontakten zur Pharmaindustrie. Vgl. etwa CAMPELL / GREUN / MOUNTFORD et al. (2007), S. 356 sowie zusammenfassend JELINEK / NATE (2009), S. 3 f.

17 FUGH-BERMAN / AHARI (2007); OLDANI (2004), S. 334, 345; Das Minimal-Ziel liegt dabei in der Markenetablierung durch „reminder items". Vgl. KATZ / CAPLAN / MERZ (2003), S. 39,40 .

18 Dazu OLDANI (2004), S. 334

19 Sehr anschaulich die Zitate, Methoden und Ärztekategorien bei FUGH-BERMAN / AHARI (2007), S. 621 f. und WEISS (2008), S. 51 f.; zu speziellen Methoden wie z.B. „spin-selling bzw. „spin-doctoring " hierbei auch OLDANI (2004), S. 328 f. (mit einem Gesamtmodell eines „pharmaceutical gift cycle“); ferner etwa KORZILIUS / RIESER (2007), S. 104.

20 Dazu m.w.N. KATZ / CAPLAN / MERZ (2003), S. 39, $40 \mathrm{f}$.
21 Vgl. die sehr anschaulichen Zitate, Methoden und Ärztekategorien bei FUGH-BERMAN / AHARI (2007), S. 621f, ferner etwa KORZILIUS / RIESER 2007, S. 104; OLDANI (2004), S. $335 \mathrm{f}$.

22 JELINEK / NEATE (2009), 3 f.; WEISS (2008), S. 51 f., 61 f.; FUGH-BERMAN / AHARI (2007), S. 624; MOYNIHAN (2003), S. 1189 führt 16 verschiedene Maßnahmen auf.

23 Hierzu am Beispiel von Onkologen CHREN / LANDEFELD (1992), S. 688.

24 So schon BOWMANN / PEARLE (1998), S. 15; LEXCHIN (1993), S. $1401 \mathrm{f}$.

25 Vgl. etwa LIEB / BRANDTÖNIES (2010), S. 395.

26 Vgl. die Meta-Analyse bei SCHNEIDER / LÜCKMANN (2008), S. 516 f.; für Deutschland im Detail LIEB / BRANDTÖNIES (2010), S. 395; Im Bezug auf Kollegen etwa international etwa RANDALL / ROSENBAUM / ROHRBAUGH et al. (2005), S. 38 m.w.N.; Als ethisch problematisch werden die Zuwendungen dabei nicht eingestuft vgl. BRETT / BURR / MOLOO (2003), S. 2216.

27 Vgl. PRAGAL (2005), S. 133; mit Blick auf Apotheker auch PRAGAL / APFEL (2007), S. 10 f.; KLÜMPER / EGGERTS (2009).

28 Vgl. BGH v. $25.11 .2003-4$ StR 239/03 = BGHSt 49, 17.

29 Stellvertretend und m.w.N. ULSENHEIMER (2008), S. 521 Rn. 13/41; GEIS (2005), 369 f.; zuletzt ebenso wieder z.B. SAHAN / URBAN (2011), S. 23 f.

30 Einschlägige Verfahren wurden mit Blick auf $\$$ 299 StGB ausnahmslos nach $\$ 170$ Abs. 2 StPO eingestellt. Dazu SCHNEIDER (2010), S. 242.

31 FISCHER (2011), $\mathbb{S} 299$, Rn. 10 b; TIEDEMANN (2008) in: Leipziger Kommentar StGB, \$ 299 Rn. 18; DANNECKER (2010) in: Nomos Kommentar StGB, $\mathbb{S} 299$ Rn. 22, 23c; BANNENBERG (2008) in: Nomos Handkommentar StGB, $\mathbb{2} 299$ Rn. 9.

32 Vgl. nur - jeweils m.w.N. - FRISTER (2010); KÖLBEL (2011a), S. 195 f.

33 OLG Braunschweig v. 23. 2. 2010 - Ws $17 / 10$ NStZ 2010, 392; AG Ulm v. 26.10.2010 - 3 Cs 37 Js 9933/07; LG Hamburg v. 9.12.2010 - 618 KLs 10/09. Der 3. und der 5. Strafsenat des BGH haben sich dem angeschlossen, diese Frage aber mit Beschluss vom 5.5.2011 (3 StR 458/10) bzw. 20.7.2011 (5 StR 115/11) dem Großen Senat für Strafsachen vorgelegt.

34 Hierzu zusammenfassend und m.w.N. KÖL BEL (2008b), S. $75 \mathrm{f}$.

35 Aus den zahlreichen Pressebeiträgen http:/ www.aerzteblatt.de/v4/archiv/sslcme. asp? $\mathrm{src}=\& \mathrm{id}=65930 \& \mathrm{p}$ oder http://www.faz. net/s/Rub0E9EEF84AC1E4A389A8DC6C23 161FE44/Doc EC3357310CB464242B2CBF D5C757EB2AD ATpl Ecommon Scontent. html - Aufruf jeweils v. 13.10.2011).

36 Zur Normentwicklung näher KLÜMPER (2009)

37 Zum Ganzen KLÜMPER (2009), S. 593, 595; BURK (2010), S. 89; FULDA (2010), S. 97; KAUFMANN / VOLAND (2011), S. $281 \mathrm{f}$

38 TEUBNER (1999), S. 14; ähnlich BOERS / THEILE / KARLICZEK (2003); THEILE (2008).

39 KÖLBEL (2008a), S. 76, 77.

40 PASSARGE (2011), S. 83f; KLÜMPER WALTHER (2010), S. 147

41 Zum Ganzen KLÜMPER / VOLLEBREGT (2009), S. 316, 317.

42 Vgl. stellvertretend für Bayer Schering: http:// www.bayer.de/de/corporate-compliance-policy. aspx (Aufruf v. 13.10.2011). Weiterer Überblick bei HOFER / KLÜMPER (2009), S. 23, $25 \mathrm{f}$.

43 Näher etwa KUHLEN (2010); KLÜMPER / EGGERTS (2010). 
44 Das Fallaufkommen dieser außerstaatlichen Judikatur ist marginal und liegt dauerhaft im zweistelligen Bereich (vgl. Jahresberichte unter http://www.fs-arzneimittelindustrie.de/ downloads.html - Aufruf v. 13.10..2011). Zur dortigen Judikatur näher KUHLEN (2010), S. 882 .

45 KLÜMPER / WALTHER (2010), S. 146, 147.

46 KLÜMPER / WALTHER (2010), S. 147.

47 Vgl. etwa EHLERS (2005), S. 97 f.; für die entsprechende Rechtsberatung stellvertretend PASSARGE (2011), S. 83 f. sowie das Seminarbeispiel unter http://www.ra-wigge.de/Da-
teien/Veranstaltungen/euroforum10_03_10.pdf (Aufruf v. 13.10.2011).

48 Näher zu den im Anschluss angesprochenen Entwicklungen KÖLBEL (2011b).

49 So setzen manche Strategievarianten auf Kooperationsverträge mit den Kassen und vertriebsförderliche Effekte im Rahmen der sog. Integrierten Versorgung. Der neu gefasste \ 140 b I Nr. 8 SGB V macht nämlich auch pharmazeutische Unternehmen zu möglichen Vertragspartnern der Krankenkassen. Dass in diesen Versorgungsmodellen das eigene Arzneimittelprodukt keine Rolle spielen soll, mag man nicht ernstlich glauben. Für ein Beispiel einer solchen Kooperation zwischen der AOK Niedersachsen und dem Pharmakonzern Janssen-Cilag vgl. http://www.janssen-cilag. de/news/detail.jhtml?itemname=news_company_83 (Aufruf v. 13.10.2011).

50 Etwa HARMS / GÄNSHIRT / LOSERT (2005), S. 868.

51 Vgl. GLAESKE / SCHUBERT (2006), S. 16.

52 BAUER / HAHN / HAMMERSCHMIDT (2006).

53 Vgl. etwa KHANFAR / POLEN / CLAUSON (2009), S. $451 \mathrm{f}$

\section{„Skimming“ - Eine kriminologische Betrachtung}

Mario Bachmann und Ferdinand Goeck

\section{Einleitung}

„Geldautomatenmanipulation boomt in Deutschland “1 - Diese und ähnliche Schlagzeilen sind in jüngster Zeit häufig zu lesen. Gemeint ist das sogenannte Skimming, das in den letzten zehn Jahren ebenso wie andere Formen von Kriminalität im modernen Zahlungsverkehr (z.B. Phishing/Pharming) vermehrt in den Fokus der Öffentlichkeit geraten ist. Hierbei werden durch gezielte Manipulationen an Geldautomaten Daten von Bankkarten ausgespäht, um mit den so erlangten Informationen Dubletten zur späteren Geldabhebung herzustellen. Bisher wurde das Skimming vor allem unter strafrechtsdogmatischen Gesichtspunkten erörtert. ${ }^{2}$ Kriminologische Aspekte spielten dabei allenfalls am Rande eine Rolle. Der vorliegende Beitrag will insoweit Abhilfe schaffen und nach einer kurzen Darstellung der technischen Vorgehensweise (II.) sowie einer knappen strafrechtlichen Einordnung (III.) die Phänomenologie in den Blick nehmen (IV.). Im Anschluss daran soll eine Interpretation der statistischen Befunde erfolgen (V.), bevor schließlich nach Präventionsansätzen gefragt wird (VI.).

\section{Vorgehensweise}

\section{Skimming}

Um die Vorgehensweise der Täter beim Skimming zu verstehen, ist es zunächst notwendig, sich die Funktionsweise von Zahlungskarten (= Debit- oder Kreditkarten ${ }^{3}$ ) vor Augen zu führen. So ist zu beachten, dass die für eine Transaktion erforderlichen Daten (Bankleitzahl, Kontonummer, Gültigkeitsdauer der Karte u.a.) sowohl auf dem Chip als auch auf dem Magnetstreifen der
Karte gespeichert sind, wobei die Informationen (nur) auf letzterem in unverschlüsselter Form vorliegen. ${ }^{4}$ Demgegenüber ist die Geheimzahl des Bankkunden (PIN) nicht auf dessen Zahlungskarte gespeichert, sondern wird bei der Eingabe am Geldautomaten verschlüsselt erfasst und im Regelfall mit sogenannten „PIN-Verify-Keys“ “im Rechenzentrum der Bank überprüft. ${ }^{5}$

Da Karteninformationen und Geheimzahl somit getrennt voneinander „aufbewahrt“ werden, erfolgt das Skimming regelmäßig in zwei Schritten. Zunächst müssen die Täter an die unverschlüsselten Kartendaten des Bankkunden und danach an dessen PIN gelangen. Zum Ausspähen der erstgenannten Informationen werden handelsübliche Lesegeräte verwendet, die bereits für deutlich unter 50 Euro im Handel zu erwerben sind. Diese werden als gewöhnliche Einzugsvorrichtung getarnt und auf den regulären Einzug eines Geldautomaten (oder in Einzelfällen auch auf den Türöffner der Bankfiliale) montiert. Beabsichtigt ein Kunde nunmehr Geld abzuheben, schiebt er die Zahlungskarte in das vermeintliche Einzugsfach und seine Daten werden noch vor ihrer ordnungsgemäßen Bearbeitung im Geldautomaten von den Tätern ausgelesen. Im Anschluss daran wird die Eingabe der PIN - oftmals mittels einer Miniaturkamera ${ }^{6}$ - aufgezeichnet oder per Funk direkt übertragen. ${ }^{7}$ Alternativ ist es auch möglich, die PIN mittels eines getarnten Aufsatzes auf dem eigentlichen Eingabefeld des Bankautomaten auszuspähen. ${ }^{8} \mathrm{Da}$ bei werden die Tastenanschläge des Kunden entweder in der Attrappe gespeichert oder direkt per Funk an die Täter übertragen. Das Ausspähen der Kartendaten kann mitunter auch durch einen Kassierer erfolgen, der die EC- oder Kreditkarte eines Kunden beim Bezahlvorgang an der Kasse heimlich durch ein zweites Lesegerät zieht. ${ }^{9}$ Ferner ist es möglich, Terminals im POS-Verfahren (,point of sale“) ${ }^{10}$ so zu manipulieren, dass Karteninformationen und PIN gleichzeitig ausgespäht werden können. ${ }^{11}$

Sobald den Tätern alle erforderlichen Daten vorliegen, werden diese auf Kartenrohlinge (,white plastics“) überspielt, die zusammen mit der Geheimzahl im Ausland zum Geldabheben („,cashing“) verwendet werden. ${ }^{12}$ Mitunter werden die ausgespähten Informationen nicht direkt eingesetzt, sondern über das Internet in Staaten außerhalb Europas verkauft. ${ }^{13}$ Beim sogenannten ,carding “14 werden hingegen mit den ausgepähten Daten zunächst Waren bestellt, um diese anschließend über fremde oder eigene Onlineshops weiterverkaufen zu können. In einer Vielzahl von Fällen bedienen sich die Täter zudem des „cardings on demand“. Dies bedeutet, dass der Inhaber ausgespähter Kreditkartendaten bei einer anderen Person (dem sogenannten „carder“) die gewünschte Ware bestellt und zugleich die erforderlichen Kartendaten übermittelt. Letztere verwendet der carder sodann für die eigentliche Bestellung des Produkts und erhält hierfür in der Regel zwischen $25 \%$ und $40 \%$ des Realpreises der bestellten Ware. Vorteil einer solchen arbeitsteiligen Handlungsweise dürfte aus Sicht des Bestellers sein vermindertes Entdeckungsrisiko sein, da er die Möglichkeit hat, seine Identität beim Bestellvorgang weitestgehend zu verschleiern. Der carder wiederum wird sich in der Regel auf den Einsatz missbräuchlich erlangter Kreditkarten spezialisiert haben, auf deren Beschaffung er gerade nicht angewiesen ist. 